\title{
Structure and mechanism of ABC transporters
}

\section{Stephan Wilkens}

Address: Department of Biochemistry, State University of New York Upstate Medical University, Syracuse, NY 13210, USA

Email: wilkenss@upstate.edu

Fl000Prime Reports 2015, 7:14 (doi:10.12703/P7-14)

All FI000Prime Reports articles are distributed under the terms of the Creative Commons Attribution-Non Commercial License (http://creativecommons.org/licenses/by-nc/3.0/legalcode), which permits non-commercial use, distribution, and reproduction in any medium, provided the original work is properly cited.

The electronic version of this article is the complete one and can be found at: http://fl000.com/prime/reports/b/7//4

\begin{abstract}
All living organisms depend on primary and secondary membrane transport for the supply of external nutrients and removal or sequestration of unwanted (toxic) compounds. Due to the chemical diversity of cellular molecules, it comes as no surprise that a significant part of the proteome is dedicated to the active transport of cargo across the plasma membrane or the membranes of subcellular organelles. Transport against a chemical gradient can be driven by, for example, the free energy change associated with ATP hydrolysis (primary transport), or facilitated by the potential energy of the chemical gradient of another molecule (secondary transport). Primary transporters include the rotary motor ATPases (F-, A-, and V-ATPases), P-type ATPases and a large family of integral membrane proteins referred to as "ABC" (ATP-binding cassette) transporters. ABC transporters are widespread in all forms of life and are characterized by two nucleotide-binding domains (NBD) and two transmembrane domains (TMDs). ATP hydrolysis on the NBD drives conformational changes in the TMD, resulting in alternating access from inside and outside of the cell for unidirectional transport across the lipid bilayer. Common to all $A B C$ transporters is a signature sequence or motif, LSGGQ, that is involved in nucleotide binding. Both importing and exporting $A B C$ transporters are found in bacteria, whereas the majority of eukaryotic family members function in the direction of export. Recent progress with the X-ray crystal structure determination of a variety of bacterial and eukaryotic $A B C$ transporters has helped to advance our understanding of the ATP hydrolysis-driven transport mechanism but has also illustrated the large structural and functional diversity within the family.
\end{abstract}

\section{Introduction}

The transport of organic and inorganic molecules across cellular membranes is vital to all forms of life, as it allows cells to maintain an off equilibrium condition. In Escherichia coli, for example, $10 \%$ of the entire genome is dedicated to membrane-bound and soluble proteins involved in transport processes [1]. On the timescale relevant for cellular metabolism, the lipid bilayer represents a formidable barrier for most charged and polar molecules while allowing for the passage of hydrophobic organic compounds by passive diffusion [2]. Transport against a chemical gradient (e.g. import of nutrients) requires a source of free energy, either provided by the potential energy of an existing chemical gradient or a coupled enzymatic reaction. Transporters that are driven by the chemical gradient of a "helper" molecule are referred to as secondary transporters, while transporters that generate the driving force by an enzymatic reaction with a "high energy" molecule (mostly ATP) are called primary transporters (see the Transporter Classification Database [www.tcdb.org] for details) [3]. Transporters that use ATP hydrolysis to pump molecules across the membrane are referred to as transport ATPases, a large superfamily that includes the rotary motor F-, A-, and $\mathrm{V}$-ATPases, the P-type ATPases and the ABC transporters [4]. While transport substrates of the rotary motor and P-type ATPases are, with few exceptions, limited to protons or metal ions, $\mathrm{ABC}$ transporters cover a wide spectrum of substrates, from small inorganic and organic molecules, such as amino acids, sugars, nucleosides, vitamins and metal clusters to larger organic compounds, including peptides, lipid molecules, oligonucleotides and polysaccharides. Over the past decade, several moderateto-high resolution crystal structures have been solved for 
a variety of $\mathrm{ABC}$ transporters from microorganisms and higher eukaryotes, including mammals. The structural data, together with sophisticated biochemical and biophysical studies, have provided a wealth of information on the catalytic mechanism of ATP hydrolysis-driven transport. This mini-review gives a brief overview of the current understanding of the structure and mechanism of $\mathrm{ABC}$ transporters and what some of the remaining and emerging questions are.

\section{The ABC transporter family}

Early biochemical studies on bacterial nutrient import systems revealed a class of multi-subunit transporters that all contained an essential cytoplasmic factor with ATP hydrolysis activity (reviewed in [5]). As more amino acid sequence information became available, it was recognized that the primary structure of the ATP-binding domains of these transporters was highly conserved, including the presence of a phosphate-binding loop (P-loop or Walker A motif) and a short consensus sequence "LSGGQ". The family of transporters was subsequently termed $\mathrm{ABC}$ transporters in recognition of the "cassette-like" nature of the ATP-binding subunit [6]. Around the same time, biochemical studies on the mammalian multi-drug resistance (MDR) export pump P-glycoprotein revealed the presence of the very same motifs in its ATP-binding domain, demonstrating that the family of $A B C$ transporters was represented not only in bacteria but also higher eukaryotes, including mammals. From the current sequence information of microbial genomes, $\mathrm{ABC}$ transporters represent the largest protein family identified to date, highlighted by the fact that between 1 and 3\% of bacterial and archaeal genomes encode for subunits of $\mathrm{ABC}$ transporters [7]. There are 48 $\mathrm{ABC}$ transporters in human $[8,9]$ and many of these have been shown to be responsible for or involved in disease states, including cystic fibrosis, Tangier disease, adrenoleukodystrophy, and cancer (see below).

\section{General architecture of $A B C$ transporters}

$\mathrm{ABC}$ transporters classified so far can be grouped into exporters and importers with the importers further divided into two classes (I and II), depending on details of their architecture and mechanism [10-12]. The related family of energy-coupling factor (ECF) transporters $[13,14]$ (sometimes referred to as class III ABC importers) is structurally and functionally more distinct $[15,16]$, and this class will not be discussed here. While bacteria employ both $\mathrm{ABC}$ importers and exporters, eukaryotes, with very few exceptions, only have exporters. The canonical $\mathrm{ABC}$ transporter is organized in four functional units or domains, two NBDs (NBD1, NBD2) and two TMDs (TMD1, TMD2). In bacteria, the four domains can be a combination of individual, pairwise identical subunits, or a combination of fused NBDs and/or TMDs [12]. In eukaryotes, the majority of $\mathrm{ABC}$ transporters are constituted by a single polypeptide that contains all four functional units, with some members assembled from "half" transporters with either identical (homodimeric) or different (heterodimeric) halves. Besides the four main domains, bacterial importers require an accessory subunit that is responsible for capturing transport substrate (solute) and delivering it to the binding site in the TMDs. In Gramnegative bacteria, the accessory subunits are $30-50 \mathrm{kDa}$ soluble proteins that are found in the periplasm, while in Gram-positive microorganisms, the accessory subunits are lipoproteins anchored to the outer leaflet of the plasma membrane. Figure 1 gives an overview of some of the prominent $\mathrm{ABC}$ transporter family members for which high-resolution structural information is available.

\section{Structure and properties of the NBDs}

Thanks to its high level of similarity and the presence of several conserved motifs, the NBD or ATP-binding cassette is the hallmark of the $\mathrm{ABC}$ transporter family. Sequence identity for the NBDs within and between bacterial and eukaryotic exporters is high, with values between 30 and 50\%, pointing to a similar threedimensional fold and a conserved mechanism of energy coupling. The conserved nature of the tertiary structure can be seen in X-ray crystal structures of isolated NBDs and NBDs that are part of intact transporters. Early structures of isolated NBDs, for example, the histidine permease [17], Rad50 [18] (a DNA repair protein), the archaeal ABC transporter MJ0796 [19], and the maltose transporter [20], revealed the presence of a RecA-like domain with a Rossman fold and Walker A motif typical for NBDs, as well as an $\alpha$-helical domain that contains the signature sequence LSGGQ that is characteristic for the NBDs of $\mathrm{ABC}$ transporters. These crystal structures of isolated NBDs bound to ATP showed the two NBDs engaged in a symmetric dimer with the two ATP molecules sandwiched in the dimer interface (except for the histidine permease NBDs, which crystallized as a non-physiological dimer [17]). In this so-called "sandwich dimer", ATP is bound to NBD1 coordinated by P-loop residues from NBD1 and from residues of the signature sequence of NBD2 and vice versa for the second ATP (see Figure 1D). The ATP-bound sandwich dimer, together with the observation that nucleotide-free NBDs often crystallized as monomers or non-physiological dimers [21], suggested early on that the nucleotide-dependent dimerization of the NBDs is part of the "power stroke" driving conformational changes in the TMDs [20].

\section{Properties of the TMDs}

Depending on the transporter class, each TMD has 6 to 10 transmembrane $\alpha$-helices (with most exporters having 6) 
Figure I. Structural features of ABC transporters

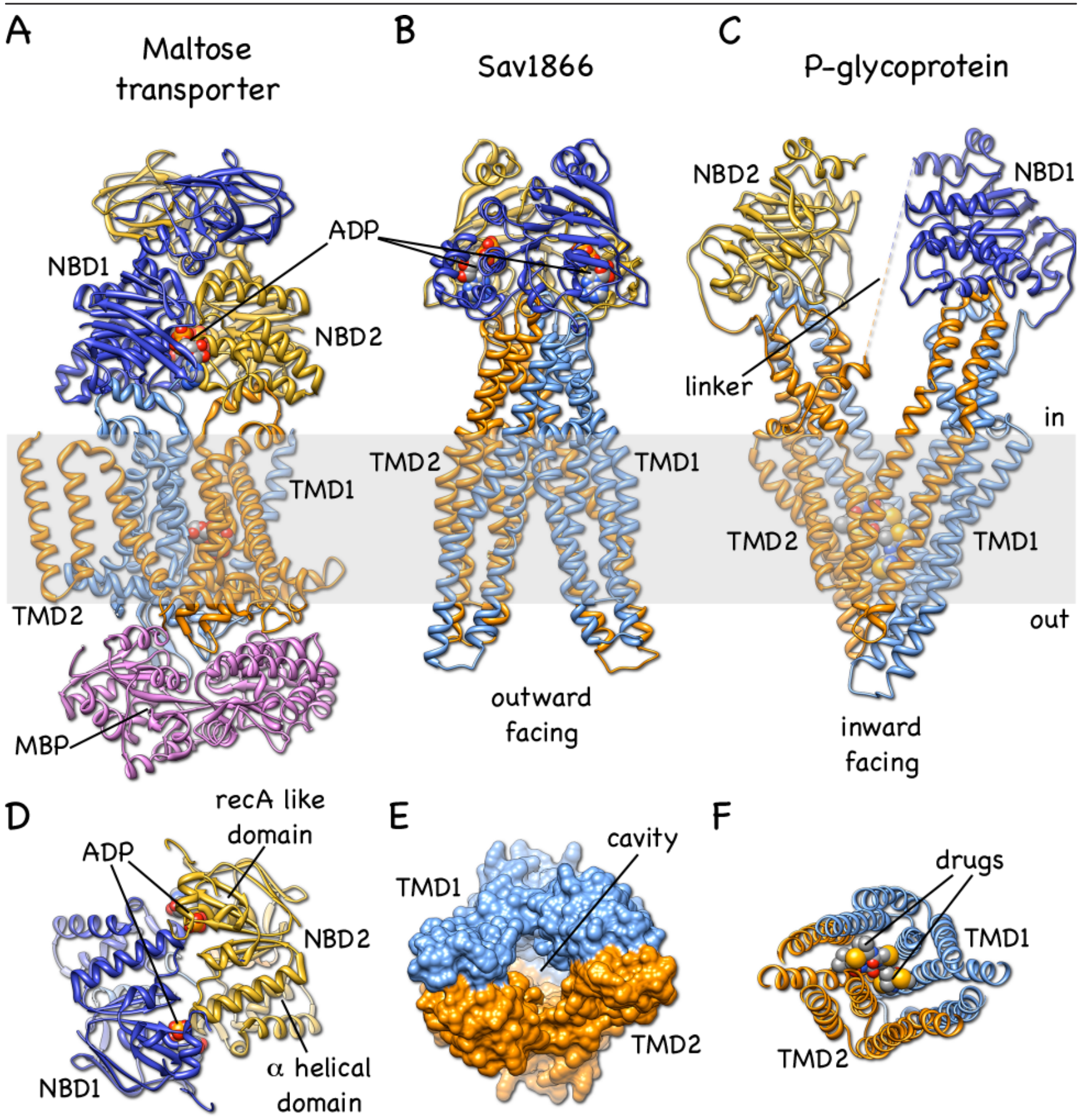

(A) Outward-facing maltose transporter with $\mathrm{ADP} \cdot \mathrm{VO}_{4}$ in catalytic sites and maltose bound to the transmembrane domain ([TMD] 3puv.pdb) [23].

(B) Homodimeric exporter Savl 866 from Staphylococcus aureus in the outward-facing conformation with ADP in catalytic sites (2hyd.pdb) [37].

(C) P-glycoprotein in the inward-facing conformation with an inhibitor molecule bound at the TMDs (4m2t.pdb) [27].

(D) The nucleotide-binding domain (NBD) sandwich dimer of the maltose transporter (MalK) as seen from the cytoplasmic side.

(E) The cavity formed by the TMDs of outward-facing Sav1866. Note that the cavity does not provide access to the outer leaflet of the lipid bilayer.

(F) Cross-section through the TMDs of glycoprotein showing the two inhibitor molecules.

ABC, ATP-binding cassette; MRP, multidrug resistance associated protein; NBD, nucleotide-binding domain; TMD, transmembrane domain. 
for a total of 12-20 transmembrane segments for a full transporter, respectively $[10,11]$. The transmembrane $\alpha$-helices of the two TMDs are packed in such a way that they form a transmembrane pore that is either accessible from the cytoplasm (inward facing; Figure 1C) or the outside of the cell (outward facing; Figure 1B). Unlike the NBDs, the TMDs generally display no significant sequence conservation but share a similar topology within a transporter class. The lack of primary structure conservation in the TMDs is likely due to the diverse nature of the transport substrates. Sequence conservation can be high between TMD1 and TMD2 in eukaryotic single-polypeptide transporters (e.g. 30\% in P-glycoprotein), suggesting that the two TMDs in these transporters are a result of gene duplication originating from homodimeric ancestors. For both importers and exporters, transport substrate has to interact at one point or another with residues of the transmembrane $\alpha$-helices that line the transmembrane pore. For bacterial type I importers (e.g. the maltose importer from E. coli), specific residues in the TMD that are involved in substrate binding have been identified from crystallographic studies and mutagenesis experiments [22,23]. For the $E$. coli vitamin B12 importer (BtuCDF, a type II importer), the translocation path does not seem to provide a specific substrate binding site but there is a hydrophobic cavity mid-membrane that can be blocked from both sides of the bilayer [24]. The situation is again different in the multidrug transporter P-glycoprotein, where several overlapping drug-binding sites have been identified [25]. The drug-binding pocket of P-glycoprotein has therefore been described as having "polyspecificity" towards its transport substrates [26-28].

\section{Mechanism of ABC transporters}

With few exceptions, $A B C$ transporters have to pump transport substrates against a chemical gradient, a process that requires ATP hydrolysis as a driving force. Under physiological conditions, $\mathrm{ABC}$ transporters operate in a single direction (either import or export), although the drug efflux pump LmrA has been shown to be reversible under certain conditions [29], which means that the membrane domain must operate one or more "turnstilelike" gates that are tightly coupled to the catalytic cycle on the NBDs. To satisfy this condition, the transmembrane domain alternates between outward- and inward-facing conformations, reminiscent of the mechanism originally proposed by Jardetzky for the P-type ATPases [30]. The mechanism is also employed by the major facilitator superfamily (MFS) of secondary transporters, in which case the driving force is provided by the potential energy of the chemical gradient of a "secondary" transport substrate, for example, protons or sodium ions [31]. In the case of $\mathrm{ABC}$ transporters, conformational switching of the membrane domain for providing alternating access is driven by the binding of transport substrate and MgATP, followed by ATP hydrolysis and product release. Based on structural and biochemical data, several models of $A B C$ transporter mechanisms have been proposed, most notably the "alternating site" [32], "switch" [33], and "constant contact" [34,35] models. While all these models share elementary steps, such as ATP-dependent NBD dimerization and the switching of the TMD between outward- and inward-facing conformations, the models diverge with respect to some of the details of the mechanism. However, it should be pointed out that there is little evidence to suggest that all $\mathrm{ABC}$ transporters function by the very same mechanism. Among the structurally and mechanistically best-characterized importers are the E. coli maltose $[22,23]$ (a type I) and vitamin B12 [24,36,37] (a type II) uptake systems. For the exporters, a large amount of biochemical and structural data are available for the multidrug resistance pumps from Staphylococcus aureus (Sav1866) [38] and higher eukaryotes (P-glycoprotein, ABCB1) [27,39-41], multidrug resistance-associated protein (MRP1, ABCC1) [42], the bacterial lipid flippase MsbA [43], and the transporter involved in antigen processing (TAP) [44].

\section{The catalytic cycle}

The basic catalytic cycle of $\mathrm{ABC}$ transporters starting from the "apo" or ground state consists of a series of steps. These include the binding of substrate-binding proteins (for importers) or the direct binding of a substrate (for exporters) to the TMDs, binding of two MgATP molecules to the NBDs, dimerization of the NBDs, switching of the TMDs between the in- and outward or out- and inward-facing conformations (depending on transporter type), ATP hydrolysis, phosphate, ADP and transport substrate release concomitant with NBD dissociation to reset the transporter to the ground state for the next cycle. The details and order of these steps depend, to some extent, on the transporter type, as illustrated in Figure 2A for exporters, Figure 2B for type I importers, and Figure 2C for type II importers. However, while there is general agreement that all or some of the above steps must happen at some point during the cycle, there is much less understanding as to the exact order of these steps and which step of the ATP hydrolysis reaction on the NBDs provides the "power stroke" that resets the TMDs to the ground state. According to the switch model [33], which was inspired by biochemical studies and early crystal structures that showed (on the one hand) ATP-dependent dimerization of isolated NBDs [20] and (on the other hand) intact apo (ATP and substrate-free) transporters in which the NBDs were seen far apart $[26,27,43]$ (see Figure 1D and C, respectively), the NBDs have to dissociate completely for product release and the 
Figure 2. Schematic of the mechanism of $A B C$ exporters and importers
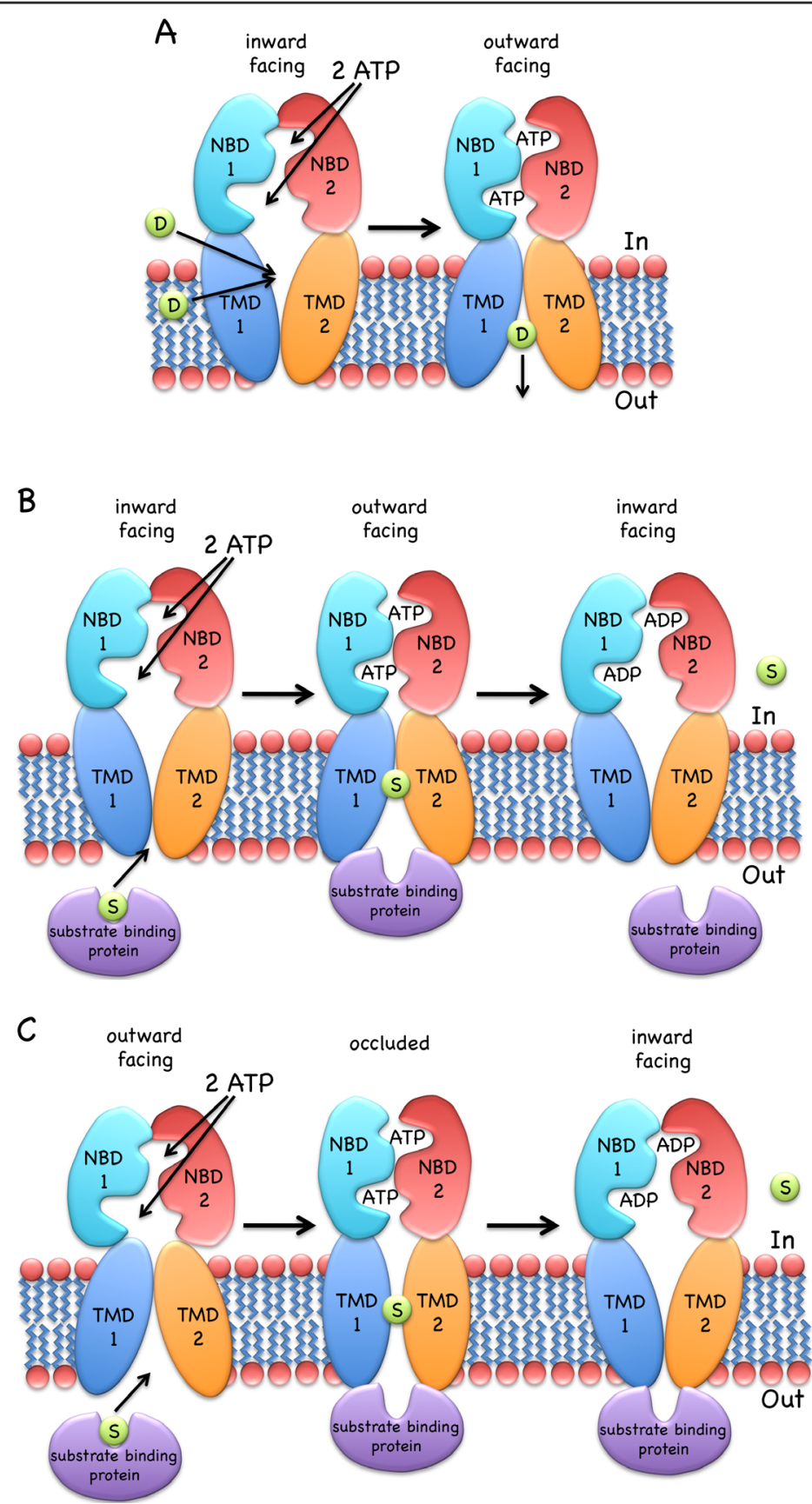

(A) The inward-facing exporter binds substrate "D" (drug) from the cytoplasm or the inner leaflet of the bilayer. After binding two molecules of MgATP, the nucleotide-binding domains (NBDs) dimerize and switch the transmembrane domain (TMDs) from the inward- to the outward-facing conformation, followed by the release of the drug to the extracellular milieu. ATP hydrolysis, ADP/Pi release and NBD dissociation resets the transporter to the inward-facing conformation. Note that there are likely intermediate conformations, some asymmetric, that have not yet been resolved by crystallography. (B) The inward-facing type I transporter (e.g., MalFGK 2 ) binds to the substrate containing periplasmic binding protein and two molecules of MgATP. NBDs dimerize and result in the outward-facing conformation. Substrate leaves the binding protein and binds to the TMDs mid-membrane. ATP is hydrolyzed and product release, together with NBD dissociation, resets the transporter to the inward-facing conformation.

(C) The outward facing type II importer (e.g., BtuCD) binds to substrate binding protein and two molecules of MgATP. Dimerization of the NBDs results in the occluded conformation with substrate confined to a sealed cavity mid-membrane. Subsequent ATP hydrolysis and NBD dissociation allows substrate to escape into the cytoplasm. A fourth, asymmetric, conformation as seen for BtuCDF is not shown.

Abbreviations: ABC, ATP-binding cassette; NBD, nucleotide-binding domain; TMD, transmembrane domain. 
start of a new cycle. However, there is experimental evidence that the NBDs operate in an alternating fashion [32], an observation that is difficult to reconcile with the simple switch model. Alternating ATP hydrolysis as well as occlusion of non-hydrolyzable $\operatorname{ATP} \gamma S$ at one catalytic site $[34,35]$ and drug-stimulated ATPase activity of P-glycoprotein, in which NBDs were covalently linked via disulfide bond [45], are all consistent with the constant-contact model in which NBDs remain associated during steady-state turnover to allow for sequential ATP hydrolysis. A "reciprocating twin-channel" model of transport that incorporates the constant-contact model has recently been proposed [46]. High-resolution crystal structures of the maltose transporter in pre-hydrolysis and transition state conformations showed that ATP hydrolysis is base catalyzed by a glutamate residue at the end of the Walker B motif that, together with assistance from residues in other conserved motifs (D-, Q-, and H-loops) polarizes a water molecule for the attack on the $\gamma$-phosphate [23]. Since the residues involved in ATP hydrolysis are highly conserved, it is reasonable to assume that this part of the mechanism is conserved in the $\mathrm{ABC}$ transporter family. Curiously, the pre-hydrolysis and transition state conformations were very similar, suggesting that it is product release (likely phosphate) rather than the ATP hydrolysis step itself that leads to the structural changes in the NBDs that are coupled to the conformational rearrangements in the TMDs. Phosphate release is also part of the power stroke that drives the rotation of the central rotor in the rotary motor F-ATPases [46]. A key structural element for coupling NBD to TMD events is the Q-loop (named so for a conserved glutamine residue) and the so-called "coupling helix" at the NBDTMD interface $[36,38,48]$. Crystallographic and EPR spectroscopy experiments have shown that catalysis involves a rotational movement of the RecA-like domain with respect to the NBD helical domain $[49,50]$, and that this motion is likely transferred to the TMDs by the Q-loop and the coupling helix.

\section{$A B C$ transporters in human disease}

There are $48 \mathrm{ABC}$ transporters in humans that can be subdivided by phylogenetic analysis into seven distinct subfamilies A-G [8,9]. Mammalian ABC transporters are involved in the cellular export of several groups of molecules, including cholesterol and sterols, lipids, retinoic acid derivatives, bile acid, iron, nucleosides, and peptides. The essential nature of these functions is highlighted by the fact that defects in the associated transporters have been observed in a number of genetic conditions, including Tangier (ABCA1) and Stargardt (ABCA4) disease [51], immune deficiency and cancer (ABCB2/3; TAP transporter) [52], cystic fibrosis (cystic fibrosis transmembrane conductance regulator [CFTR];
ABCC7) [53], and adrenoleukodystrophy (ABCD1) [5], to name only a few. Another prominent group of human $\mathrm{ABC}$ transporters are found in the liver, placenta and blood brain barrier where they are involved in the detoxification of hydrophobic organic molecules [54]. The group includes P-glycoprotein (ABCB1), one of the best-studied ABC transporters, the MRP (ABCC1) and ABCG2. These transporters, when found highly expressed in the plasma membrane of tumor cells, can result in the failure of chemotherapy by protecting the cancer cells from the cytotoxic drugs used to fight the disease. Much effort has been spent on identifying selective inhibitors for these MDR transporters and while many compounds have been identified that inhibit P-glycoprotein function in, for example, human cell culture, no broadly applicable inhibitor is in use as of yet, due to significant side effects of the compounds [55].

\section{Non-canonical ABC transporters}

While the majority of $\mathrm{ABC}$ transporters characterized so far are just that, membrane transporters, there are a number of family members that have evolved to perform different functions. CFTR, for example, is a chloride channel, and gating of the channel is regulated by the nucleotide content of the NBDs [53]. CFTR also belongs to a class of $\mathrm{ABC}$ transporters in which one of the nucleotide-binding sites is "degenerate", resulting in a catalytic site that is still able to bind but not hydrolyze ATP efficiently. Another atypical ABC "transporter" with one degenerate ATP-binding site is the sulfonylurea receptor (SUR; ABCC8/9) [56]. SUR forms a large tetrameric complex with an inward-rectifying potassium channel $\left(\mathrm{K}_{\mathrm{ATP}}\right)$, and it has been proposed that the $\mathrm{ABC}$ transporter in this complex functions in regulation of the activity of the channel by sensing cellular ATP levels.

\section{Unresolved questions: the to-do list}

Despite the recent progress with understanding $\mathrm{ABC}$ transporter mechanism, many questions remain as to some of the details of the catalytic cycle, including how many ATPs are hydrolyzed per transport event and which step of the hydrolysis cycle provides the power stroke, whether ATP hydrolysis in one NBD is sufficient for transport, and whether NBDs remain associated during transport, to name only a few. However, as pointed out earlier, some of these questions may have different answers depending on the nature of the transporter. While we now have a good collection of crystal structures, additional structures of catalytic intermediates will be needed for a more complete understanding of the transport cycle, especially for the class of exporters. Of course, the high-resolution structures will have to be complemented by biochemical and biophysical studies that address the kinetics and dynamics of the transporters. 
From studies with the MDR pump P-glycoprotein, for example, it was shown that mutating catalytic residues or trapping ADP-vanadate in only one NBD abolished ATPase and transport activity completely, an observation that leads to the proposal of the alternating site mechanism in which the two catalytic sites hydrolyze ATP in an alternating fashion [32]. Support for such a mechanism was provided by experiments that showed that the non-hydrolyzable ATP analog $\operatorname{ATP} \gamma S$ can be stably trapped in one catalytic site, leading to an "occluded" nucleotide state [34,35]. However, most of the crystal structures of $\mathrm{ABC}$ transporters or isolated NBDs show symmetric occupancy of the NBDs with either nucleotides alone or transition state analogs bound, and so the question of whether nucleotides are hydrolyzed simultaneously, or in a specific order, remains to be determined. One approach for obtaining real-time mechanistic information is, for example, Förster resonance energy transfer (FRET) spectroscopy that allows monitoring of ligand-dependent structural changes during the active turnover of single transporter molecules $[57,58]$. These studies already showed that the ABC transporter P-glycoprotein is a highly dynamic molecule with rapidly fluctuating NBDs. Performing measurements with immobilized or optically trapped molecules for longer observation times, and including fluorescent transport substrate for three-color FRET experiments, will make it possible to delineate and define the individual steps of the $\mathrm{ABC}$ transporter catalytic cycle.

\section{Abbreviations}

ABC, ATP-binding cassette; CFTR, cystic fibrosis transmembrane conductance regulator; FRET, Förster resonance energy transfer, MDR, multidrug resistance; MRP, multidrug resistance-associated protein; NBD, nucleotidebinding domain; SUR, sulfonylurea receptor; TMD, transmembrane domain.

\section{Disclosures}

The author declares that he has no disclosures.

\section{Acknowledgments}

Dr. Rebecca Oot is gratefully acknowledged for her critical reading of the manuscript.

\section{References}

I. Blattner FR, Plunkett G, Bloch CA, Perna NT, Burland V, Riley M, Collado-Vides J, Glasner JD, Rode CK, Mayhew GF, Gregor J, Davis NW, Kirkpatrick HA, Goeden MA, Rose DJ, Mau B, Shao Y: The complete genome sequence of Escherichia coli K-I2. Science (New York, N.Y.) 1997, 277:|453-62.

2. Paula S, Volkov AG, Van Hoek AN, Haines TH, Deamer DW: Permeation of protons, potassium ions, and small polar molecules through phospholipid bilayers as a function of membrane thickness. Biophysical journal 1996, 70:339-48.
3. Saier $\mathrm{MH}$, Reddy VS, Tamang DG, Västermark A: The transporter classification database. Nucleic acids research 20|4, 42:D25I-8.

4. Pedersen PL: Transport ATPases into the year 2008: a brief overview related to types, structures, functions and roles in health and disease. Journal of bioenergetics and biomembranes 2007, 39:349-55.

5. Higgins CF: ABC transporters: from microorganisms to man. Annual review of cell biology 1992, 8:67-II3.

6. Higgins CF, Hiles ID, Salmond GP, Gill DR, Downie JA, Evans IJ, Holland IB, Gray L, Buckel SD, Bell AW: A family of related ATP. binding subunits coupled to many distinct biological processes in bacteria. Nature 1986, 323:448-50.

7. Tomii K, Kanehisa M: A comparative analysis of ABC transporters in complete microbial genomes. Genome research 1998 , 8:1048-59.

FlOOOPrime RECOMMENDED

8. Dean M, Rzhetsky A, Allikmets R: The human ATP-binding cassette (ABC) transporter superfamily. Genome research 2001 , I I:I I56-66.

9. Vasiliou V, Vasiliou K, Nebert DW: Human ATP-binding cassette (ABC) transporter family. Human genomics 2009, 3:28I-90.

10. Rees DC, Johnson E, Lewinson O: $\mathbf{A B C}$ transporters: the power to change. Nature reviews. Molecular cell biology 2009, 10:218-27.

II. ter Beek J, Guskov A, Slotboom DJ: Structural diversity of ABC transporters. The Journal of general physiology 2014, I43:419-35.

12. Holland IB, Blight MA: ABC-ATPases, adaptable energy generators fuelling transmembrane movement of a variety of molecules in organisms from bacteria to humans. Journal of molecular biology 1999, 293:381-99.

13. Erkens GB, Berntsson RP, Fulyani F, Majsnerowska M, Vujičić-Žagar A, ter Beek J, Poolman B, Slotboom DJ: The structural basis of modularity in ECF-type ABC transporters. Nature structural \& molecular biology 20I I, I 8:755-60.

\section{FlOOPrime}

\section{RECOMMENDED}

14. Eitinger T, Rodionov DA, Grote M, Schneider E: Canonical and ECF-type ATP-binding cassette importers in prokaryotes: diversity in modular organization and cellular functions. FEMS microbiology reviews 2011, 35:3-67.

15. Xu K, Zhang M, Zhao Q, Yu F, Guo H, Wang C, He F, Ding J, Zhang P: Crystal structure of a folate energy-coupling factor transporter from Lactobacillus brevis. Nature 2013, 497:268-7I.

\section{FlOOOPrime} RECOMMENDED

16. Wang T, Fu G, Pan X, Wu J, Gong X, Wang J, Shi Y: Structure of a bacterial energy-coupling factor transporter. Nature 2013, 497:272-6.

\section{FlOOOPrime}

RECOMMENDED

17. Hung LW, Wang IX, Nikaido K, Liu PQ, Ames GF, Kim SH: Crystal structure of the ATP-binding subunit of an ABC transporter. Nature 1998, 396:703-7.

\section{FlOOOPrime}

RECOMMENDED

18. Hopfner KP, Karcher A, Shin DS, Craig L, Arthur LM, Carney JP, Tainer JA: Structural biology of Rad50 ATPase: ATP-driven conformational control in DNA double-strand break repair and the ABC-ATPase superfamily. Cell 2000, I 0 I:789-800.

FlOOOPrime RECOMMENDED

19. Smith PC, Karpowich N, Millen L, Moody JE, Rosen J, Thomas PJ, Hunt JF: ATP binding to the motor domain from an ABC 
transporter drives formation of a nucleotide sandwich dimer. Molecular cell 2002, 10:139-49.

\section{FlOOOPrime}

20. Chen J, Lu G, Lin J, Davidson AL, Quiocho FA: A tweezers-like motion of the ATP-binding cassette dimer in an ABC transport cycle. Molecular cell 2003, I2:65 I-6I.

\section{FlOOOPrime}

\section{RECOMMENDED}

21. Kerr ID: Structure and association of ATP-binding cassette transporter nucleotide-binding domains. Biochimica et biophysica acta 2002, I56 I:47-64.

22. Chen J: Molecular mechanism of the Escherichia coli maltose transporter. Current opinion in structural biology 2013, 23:492-8.

23. Oldham ML, Chen J: Snapshots of the maltose transporter during ATP hydrolysis. Proceedings of the National Academy of Sciences of the United States of America 201 I, I 08:I5152-6.

\section{FlOOOPrime}

\section{RECOMMENDED}

24. Korkhov VM, Mireku SA, Locher KP: Structure of AMP.PNP. bound vitamin BI2 transporter BtuCD-F. Nature 2012, 490:367-72.

\section{FlOOOPrime}

\section{RECOMMENDED}

25. Loo TW, Bartlett MC, Clarke DM: Simultaneous binding of two different drugs in the binding pocket of the human multidrug resistance P-glycoprotein. The Journal of biological chemistry 2003, 278:39706-I0.

\section{FlOOOPrime}

\section{RECOMMENDED}

26. Aller SG, Yu J, Ward A, Weng Y, Chittaboina S, Zhuo R, Harrell PM, Trinh YT, Zhang Q, Urbatsch IL, Chang G: Structure of P. glycoprotein reveals a molecular basis for poly-specific drug binding. Science (New York, N.Y.) 2009, 323:1718-22.

\section{FlOOOPrime} RECOMMENDED

27. Li J, Jaimes KF, Aller SG: Refined structures of mouse Pglycoprotein. Protein science: a publication of the Protein Society 2014, 23:34-46.

\section{FlOOOPrime}

RECOMMENDED

28. Gutmann, Daniel AP, Ward A, Urbatsch IL, Chang G, van Veen, Hendrik W: Understanding polyspecificity of multidrug ABC transporters: closing in on the gaps in ABCBI. Trends in biochemical sciences 2010, 35:36-42

29. Balakrishnan L, Venter $H$, Shilling RA, van Veen, Hendrik W: Reversible transport by the ATP-binding cassette multidrug export pump LmrA: ATP synthesis at the expense of downhill ethidium uptake. The Journal of biological chemistry 2004, 279: I 1273-80.

\section{FlOOOPrime
RECOMMENDED}

30. Jardetzky O: Simple allosteric model for membrane pumps. Nature 1966, 21 I:969-70.

31. Reddy VS, Shlykov MA, Castillo R, Sun El, Saier MH: The major facilitator superfamily (MFS) revisited. The FEBS journal 2012, 279:2022-35

32. Senior AE, al-Shawi MK, Urbatsch IL: The catalytic cycle of P-glycoprotein. FEBS letters 1995, 377:285-9.

33. Higgins CF, Linton $\mathrm{KJ}$ : The ATP switch model for ABC transporters. Nature structural \& molecular biology 2004, I I:9|8-26.

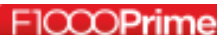

34. Sauna ZE, Kim I, Nandigama K, Kopp S, Chiba P, Ambudkar SV: Catalytic cycle of ATP hydrolysis by P-glycoprotein: evidence for formation of the E.S reaction intermediate with ATP. gamma-S, a nonhydrolyzable analogue of ATP. Biochemistry 2007, 46: 13787-99.

\section{FlOOOPrime}

RECOMMENDED

35. Siarheyeva A, Liu R, Sharom FJ: Characterization of an asymmetric occluded state of P-glycoprotein with two bound nucleotides: implications for catalysis. The Journal of biological chemistry 2010, 285:7575-86.

\section{FlOOOPrime \\ RECOMMENDED}

36. Locher KP, Lee AT, Rees DC: The E. coli BtuCD structure: a framework for ABC transporter architecture and mechanism. Science (New York, N.Y.) 2002, 296:109I-8.

\section{FlOOOPRIM
RECOMMENDED}

37. Lewinson $\mathrm{O}$, Lee $\mathrm{AT}$, Locher KP, Rees DC: A distinct mechanism for the ABC transporter BtuCD-BtuF revealed by the dynamics of complex formation. Nature structural \& molecular biology 2010, 17:332-8

\section{FIOOOPrime
RECOMMENDED}

38. Dawson, Roger JP, Locher KP: Structure of a bacterial multidrug ABC transporter. Nature 2006, 443:|80-5.

\section{FlOOOPrime}

\section{RECOMMENDED}

39. Sharom FJ: The P-glycoprotein multidrug transporter. Essays in biochemistry 2011, 50:161-78.

40. Jin MS, Oldham ML, Zhang Q, Chen J: Crystal structure of the multidrug transporter P-glycoprotein from Caenorhabditis elegans. Nature 2012, 490:566-9.

41. Loo TW, Clarke DM: Recent progress in understanding the mechanism of P-glycoprotein-mediated drug efflux. The Journal of membrane biology 2005, 206:173-85.

42. Cole, Susan PC: Targeting multidrug resistance protein I (MRPI, ABCCI): past, present, and future. Annual review of pharmacology and toxicology 2014, 54:95-II7.

43. Ward A, Reyes CL, Yu J, Roth CB, Chang G: Flexibility in the ABC transporter MsbA: Alternating access with a twist. Proceedings of the National Academy of Sciences of the United States of America 2007, 104:19005-10.

44. Abele R, Tampé R: Peptide trafficking and translocation across membranes in cellular signaling and self-defense strategies. Current opinion in cell biology 2009, $21: 508-15$.

45. Verhalen B, Wilkens S: P-glycoprotein retains drug-stimulated ATPase activity upon covalent linkage of the two nucleotide binding domains at their C-terminal ends. The Journal of biological chemistry 201 I, 286:10476-82.

46. Jones PM, George AM: A reciprocating twin-channel model for ABC transporters. Quarterly reviews of biophysics 2014, 47:189-220.

\section{FlOOOPrime} RECOMMENDED

47. Adachi K, Oiwa K, Nishizaka T, Furuike S, Noji H, Itoh H, Yoshida M, Kinosita K: Coupling of rotation and catalysis in F(I)-ATPase revealed by single-molecule imaging and manipulation. Cell 2007, 130:309-21.

FlOOOPrime

48. Hollenstein K, Frei DC, Locher KP: Structure of an ABC transporter in complex with its binding protein. Nature 2007, 446:213-6.

RECOM 
49. Khare D, Oldham ML, Orelle C, Davidson AL, Chen J: Alternating access in maltose transporter mediated by rigid-body rotations. Molecular cell 2009, 33:528-36.

\section{FlOOOPrime}

\section{RECOMMENDED}

50. Orelle C, Alvarez, Frances Joan D, Oldham ML, Orelle A, Wiley TE, Chen J, Davidson AL: Dynamics of alpha-helical subdomain rotation in the intact maltose ATP-binding cassette transporter. Proceedings of the National Academy of Sciences of the United States of America 2010, 107:20293-8.

\section{FlOOOPrime}

RECOMMENDED

51. Tarling EJ, de Aguiar Vallim, Thomas Q, Edwards PA: Role of ABC transporters in lipid transport and human disease. Trends in endocrinology and metabolism: TEM 2013, 24:342-50.

52. Leone P, Shin E, Perosa F, Vacca A, Dammacco F, Racanelli V: MHC class I antigen processing and presenting machinery: organization, function, and defects in tumor cells. Journal of the National Cancer Institute 2013, 105: 1 172-87.

53. Cant N, Pollock N, Ford RC: CFTR structure and cystic fibrosis. The international journal of biochemistry \& cell biology 2014, 52:15-25.
54. Gottesman MM, Fojo T, Bates SE: Multidrug resistance in cancer: role of ATP-dependent transporters. Nature reviews. Cancer 2002, 2:48-58.

55. Sharom FJ: ABC multidrug transporters: structure, function and role in chemoresistance. Pharmacogenomics 2008, 9:105-27.

56. Inagaki N, Gonoi T, Clement JP, Namba N, Inazawa J, Gonzalez G, Aguilar-Bryan L, Seino S, Bryan J: Reconstitution of IKATP: an inward rectifier subunit plus the sulfonylurea receptor. Science (New York, N.Y.) 1995, 270: I I66-70.

\section{FlOOOPrime}

RECOMMENDED

57. Verhalen B, Ernst S, Börsch M, Wilkens S: Dynamic ligand-induced conformational rearrangements in P-glycoprotein as probed by fluorescence resonance energy transfer spectroscopy. The Journal of biological chemistry 20I2, 287: I I 2-27.

58. Zarrabi N, Ernst S, Verhalen B, Wilkens S, Börsch M: Analyzing conformational dynamics of single P-glycoprotein transporters by Förster resonance energy transfer using hidden Markov models. Methods (San Diego, Calif.) 2014, 66:168-79. 